\title{
Measurement and calculation of the intramyocardial stress
}

\author{
S. Mihailescu \& R. M. Shoucri \\ Department of Physiology, Faculty of Medicine, \\ National Autonomous University of Mexico, Mexico City, 04510 Mexico \\ Department of Mathematics and Computer Science, \\ Royal Military College, Kingston, Ontario, Canada
}

\begin{abstract}
By using a servo-nulling mechanism and coated glass micropipettes (20-24 $\mu \mathrm{m}$ OD), intramyocardial stress (IMS) was measured at the base of the left ventricle in isolated working and nonworking cat hearts, perfused with Krebs-Henseleit buffer. Glass micropipettes were placed at various depths inside the left ventricular wall using a micropipette holder and manipulator. Calculation of the IMS is not a simple problem because of the complex structure of the active fibres in the myocardium. However a mathematical approach has been developed that reduces the complexity of this problem. It consists in using the concept of force per unit volume of the myocardium to model the components of the stress generated by the fibers in three orthogonal directions (assuming symmetrical contraction). Important relations are derived between the stress induced by the LVP and the stress induced by the active fibres in the passive medium of the myocardium.

Keywords: measurement with micropipette, intramyocardial stress, active and passive stress in the myocardium, left ventricular elastance, active force per unit volume of the myocardium, stress-strain relation in the myocardium.
\end{abstract}

\section{Introduction}

Contraction of myocardial fibers creates the hydraulic pressure necessary for displacement of blood inside the cardiovascular system. A side effect of left ventricular contraction is the compression of its coronary vessels, which results in a fasic reduction of its coronary arterial flow [1]. Systolic closure of left 
coronary arteries was attributed to an increase in pressure in the space surrounding these vessels (interstitial space). This pressure was named "tissue pressure" or "intramyocardial pressure"[2]. The accuracy of its measurement depends on the size of the transducers inserted inside the left ventricular wall, large transducers providing pressure values higher than the real ones [3]. The servo-nulling method seems the solution for this problem, because it uses as sensors glass micropipettes, which are 1000 smaller than transducers used by any other method [4]. This paper describes the use of the servo-nulling method for measuring intramyocardial pressure in the isolated beating hearts.

Various theoretical studies suggest that pressure transducers inserted inside the ventricular wall measure total intramyocardial passive stress and not intramyocardial pressure. Total intramyocardial passive stress (force/unit area) is a tensorial quantity with six components (three normal and three shear stresses), which, under the assumption of symmetric contraction of the left ventricle, can be reduced to a vectorial quantity with three components. In contrast, intramyocardial pressure is a scalar quantity. Various studies also suggest that total intramyocardial stress has both passive and active components. The passive component depends on left ventricular pressure, cavity radium and wall thickness and can be calculated according to the Laplace law. The active component is represented by the left ventricular elastance. In this study important relationships are established between intramyocardial stress induced by left ventricular pressure and the stress induced by ventricular elastance. The data derived from the mathematical model proposed for the genesis of total intramyocardial passive stress are compared with experimental data obtained using the servo-nulling system.

\section{Experimental methods}

Experiments were performed in adult cats that were anaesthetized with sodium pentobarbital $(30 \mathrm{mg} / \mathrm{kg})$ and heparinized (1000U/kg, i.v.). After thoracotomy, a polyethylene cannula was inserted into the ascending aorta and the coronary vessels were perfused at a constant pressure of $75 \mathrm{~mm} \mathrm{Hg}$, according to the Langendorff technique. The pulmonary veins were ligated, whereas the pulmonary artery was cut and left open. The hearts were removed from the thorax and placed in a perfusion system. A SPC 350 Mikro-Tip pressure transducer (Millar Instr., Houston TX) was inserted into the left ventricular cavity, through the left atrium and mitral valve. Coronary flow was collected from the pulmonary artery and re-circulated.

In nine experiments, the isolated non-working hearts were converted into working ones by inserting a cannula into the left atrium and perfusing it with Krebs-Henseleit buffer at a constant pressure of $10 \mathrm{~cm} \mathrm{H}_{2} \mathrm{O}$. The aortic flow was directed into a glass chamber half-filled with perfusate, which served as windkessel system, and from here to a vertical polyethylene tube with overflow system and adjustable height, which was used to set the afterload.

In experiments with non-working hearts $(n=9)$, the thebesian flow of the left ventricle was drained by means of a piece of polyethylene tubing inserted in this 
heart chamber through the left atrial wall and mitral valve. The aortic cannula was connected to a vertical polyethylene tube with overflow and variable height, which served to set the coronary perfusion pressure.

The perfusion fluid used was the Krebs-Henseleit buffer containing in $\mathrm{mM}$ : $\mathrm{NaCl} 118, \mathrm{KCl} 4.7, \mathrm{CaCl}_{2} 2.52, \mathrm{MgSO}_{4} 1.65, \mathrm{NaHCO} 324.88, \mathrm{KH}_{2} \mathrm{PO}_{4}$ 1.18, glucose 5.55. In order to reduce edema, dialyzed albumin was added to the perfusate in a concentration of $3 \mathrm{~g} / 100 \mathrm{ml}$. The partial pressure of oxygen and the $\mathrm{pH}$ of the perfusate were measured periodically with a blood-gas analyser and maintained at $600 \mathrm{~mm} \mathrm{Hg}$ and 7.4 respectively by equilibrating the perfusate with a gas mixture containing $95 \%$ oxygen and $5 \%$ carbon dioxide. The temperature of the perfusate was set at $37.5^{\circ} \mathrm{C}$ using a thermostat controlled water bath.

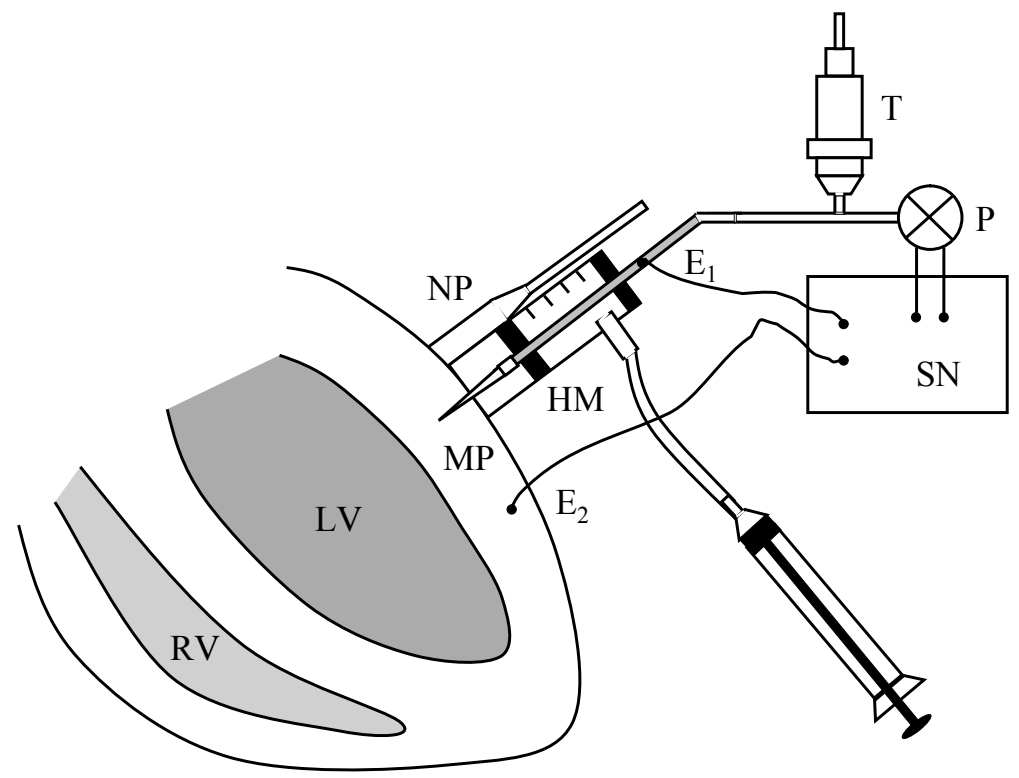

Figure 1: Setup for measuring intramyocardial stress in isolated hearts. LV, left ventricle; RV, right ventricle; MP, micropipette; NP, negative pressure; HM, holder and manipulator system; E1 and E2, electrode for measurement of impedance; $\mathrm{T}$, pressure transducer; $\mathrm{P}$, pump of the servo-nulling system ( $\mathrm{SN})$.

Measurement of intramyocardial pressure was achieved with glass micropipettes and a servo-nulling system (Instrumentation for Physiology and Medicine, San Diego, CA) (fig.1). A servo-nulling system maintains constant the electrical resistance measured between the hypertonic fluid $(\mathrm{NaCl} 1-2 \mathrm{M})$ inside a glass micropipette and the isotonic interstitial or intravascular fluids. The increase in the hydrostatic pressure outside the pipette displaces the interface between the isotonic and hypertonic fluids towards the interior of the pipette, 
which increases the electrical resistance. This activates a pressure system, which increases the hydrostatic pressure inside the pipette and thus displaces the liquid interface to its original position. The pressure inside the pipette was measured in our experiments with a Statham P23-ID transducer. The pipettes were obtained from borosilicate glass tubes (2.00 mm ID, 4 inches long) with filament inside (World Precision Instruments, FL) pulled with a Narishige type 2 puller. The diameter of the tip was in most experiments between 20 and $24 \mu \mathrm{m}$ whereas their lengths were between 7 and $9 \mathrm{~mm}$, which corresponded to the thickness of the left ventricular wall at its base. In order to increase the resistance to breakage, the pipettes were coated with a thin layer of nail polish. This procedure increased pipettes' external diameter by 1-4 $\mu \mathrm{m}$. Pipettes were filled with $\mathrm{NaCl} 1 \mathrm{M}$ and attached to a holder and micromanipulator system. This one was manufactured from the cylinder of a $1 \mathrm{ml}$ polyethylene syringe inside of which two rubber pistons were inserted. One of the rubber pieces was fixed to the cylinder whereas the other one was left mobile together with a metal tube. This last one was connected at one end with the micropipette and at the other one with the servonulling system. The space between the rubber pistons was filled with distilled water and connected to a $1 \mathrm{ml}$ syringe. Injecting small volumes of water inside this space produced the displacement of the mobile rubber piston and of the pipette for measurable distances. The holder and micromanipulator system was attached to the beating hearts using negative pressure $(-30 \mathrm{~mm} \mathrm{Hg})$ applied through a suction system attached to the cylinder's body. Impedance changes were measured between the hypertonic fluid inside the pipette and the interstitial fluid .

Pipettes were displaced inside the myocardial wall, perpendicularly on its surface, in $1 \mathrm{~mm}$ increments. At each level of myocardial depth, IMS was measured at three different coronary perfusion pressures (for non-working hearts) or afterloads (working hearts), in the interval between 50 and $100 \mathrm{~mm}$ $\mathrm{Hg}$. Integrity of the pipettes during the measurement of IMS was checked by measuring their tip's diameter before myocardial impalement and after completion of IMS measurements. Left ventricular pressure (LVP), IMS, aortic pressure and aortic flow were recorded using a CODAS (DATAQ) data acquisition system, sampling at 166 samples/s/channel.

\section{Mathematical calculation}

The mathematical model has been discussed in previous publications [5-10]. The Left ventricle is represented as a thick-walled cylinder contracting symmetrically, transverse isotropy with respect to the axis of the cylinder is assumed. A helical fiber in the myocardium is projected on its cross-section as a dotted circle as shown in fig. 2. Because of the assumed symmetry of the problem, an active force $D$ per unit volume of the myocardium is generated in the radial direction. The radial active force/unit area acting on the inner surface of the myocardium (radial fibre stress at the endocardium) is given by $\int_{a}^{b} D d r=\bar{D} h$, where $\bar{D}$ is an average value calculated by using the mean value theorem, $a=$ inner radius, $b=$ outer radius, $h=b-a=$ thickness out the 
myocardium. The equilibrium of forces at the endocardium is given by the following equation

$$
(D h)_{m}-P_{m} \approx E_{\max }\left(V_{e d}-V_{m}\right)
$$

which can be split into two equations

$$
\begin{gathered}
(D h)_{m} \approx E_{\text {max }}\left(V_{e d}-V_{d m}\right) \\
P_{m} \approx E_{\text {max }}\left(V_{m}-V_{d m}\right)
\end{gathered}
$$

where $V_{e d}$ is the end-systolic volume (when $\left.d V / d t=0\right), \quad(\bar{D} h)_{m}, P_{m}, V_{m}$ are respectively the radial active force/unit area, the ventricular pressure and the ventricular volume when the ventricular elastance $E$ reaches its maximum value $E_{\max }$ near end-systole (usually $P_{m} \approx P_{\max } / 1.2$, where $P_{\max }$ is the maximum pressure during the ejection phase). The definition of $V_{d m}$ follows from eqns (2) and (3). Usually we can take $V_{m} \approx V_{e s}$ the end-systolic volume (when $d V / d t=0$ ). The external pressure $P_{o}$ on the pericardium is neglected.

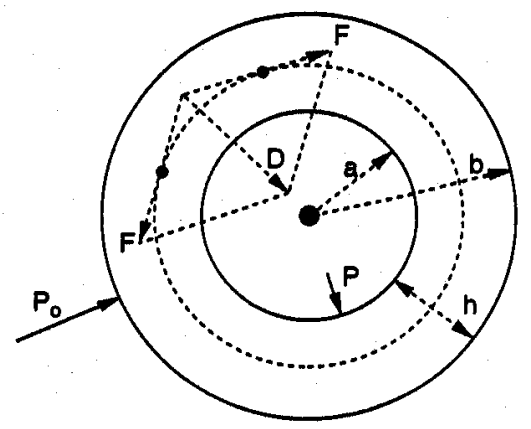

Figure 2: Cross-section of a thick-walled cylinder representing the left ventricle. The helical fibre of the myocardium is projected on the cross-section as a dotted circle. $D$ is the active radial force/unit volume of the myocardium. $P$ is the left ventricular cavity pressure, $P_{o}$ is the outer pressure on the pericardium (which is neglected). $A=$ inner radius, $b=$ outer radius, $h=$ thickness of the myocardium $=$ $b-a$.

The standard relations of linear elasticity are used to derive the stress-strain relations in the myocardium [5,11]. Although the use of linear elasticity has given contradictory results in the past [12], the mathematical approach used in this study has given consistent results. By assuming that near the equatorial region, the myocardium can be approximated by a two-dimensional model, one 
can assume that the longitudinal strain $\varepsilon_{L} \approx 0, Y_{L} \approx Y_{t}$ (Young's modulus of elasticity respectively in the longitudinal and transverse directions), and by assuming that the volume of the myocardium is constant the incompressibility condition implies that the Poisson's ratio $v_{t} \approx 0.5$. Under these assumptions and by assuming a symmetrical contraction of the myocardium, the stress components in the radial, circumferential and longitudinal directions of the myocardium are respectively given by the following equations

$$
\begin{gathered}
\sigma_{r} \approx\left(P_{m}-(\bar{D} h)_{m}\right)\left(a^{2} /\left(b^{2}-a^{2}\right)\right)\left(1-b^{2} / r^{2}\right) \\
\sigma_{c} \approx\left(P_{m}-(\bar{D} h)_{m}\right)\left(a^{2} /\left(b^{2}-a^{2}\right)\right)\left(1+b^{2} / r^{2}\right) \\
\sigma_{L} \approx\left(P_{m}-(\bar{D} h)_{m}\right)\left(a^{2} /\left(b^{2}-a^{2}\right)\right)
\end{gathered}
$$

Inner radius of the myocardium is $a$, outer radius is $b$, and the radial distance from the centre of the cylinder is $r$. The total stress is given by $\sigma_{t o t} \approx\left(\sigma_{r}^{2}+\sigma_{c}^{2}+\sigma_{L}^{2}\right)^{1 / 2}$ which gives

$$
\sigma_{t o t} \approx\left(P_{m}-(\bar{D} h)_{m}\right)\left(a^{2} /\left(b^{2}-a^{2}\right)\right)\left(3+2 b^{4} / r^{4}\right)^{1 / 2}
$$

It is clear that $\sigma_{t o t}$ can be expressed in the form $\sigma_{t o t} \approx\left(\sigma_{t o t}\right)_{p}+\left(\sigma_{t o t}\right)_{d}$, where

$$
\begin{gathered}
\left(\sigma_{t o t}\right)_{p} \approx P_{m}\left(a^{2} /\left(b^{2}-a^{2}\right)\right)\left(3+2 b^{4} / r^{4}\right)^{1 / 2} \\
\left(\sigma_{t o t}\right)_{d} \approx(-\bar{D} h)_{m}\left(a^{2} /\left(b^{2}-a^{2}\right)\right)\left(3+2 b^{4} / r^{4}\right)^{1 / 2}
\end{gathered}
$$

Similar decomposition also applies to the individual components of the stress $\sigma_{r}$, $\sigma_{c}$ and $\sigma_{L}$. The question now is what is exactly measured by the micropipette in the results reported in fig. 4A of Mihailescu and Abel [4]. The reason why we have identified the measured quantity reported in fig. 4A of [4] with $\sigma_{t o t}$ as expressed by eqn (7) is the fact that $P_{m}-(\bar{D} h)_{m}$ as calculated from eqn (7) remains constant for consecutive measurements as $r$ varies from the epicardium to the endocardium, which is what should be expected. This result is shown in Table 1 for the three-perfusion pressures PP reported in fig. 4A of [4]. In these calculations the inner radius $a=0.74 \mathrm{~cm}$ and the outer radius $b=1.54 \mathrm{~cm}$. The respective values of the left ventricular pressure $P \approx P_{\max } / 1.2$ used in the calculation are shown Table 1; all units are in mmHg. The results in fig. 3 show the graphical relations of the differences $\nabla(\bar{D} h)_{m}$ and $\nabla(\bar{D} h-P)_{m}$ calculated between pair of values at two different PP at the same radial distance $r$. Fig. 3 is an indication of the consistency of the calculations reported in Table 1. Fig 4 (left) shows the relation between the components of the stress induced in the passive medium of the myocardium by $(\bar{D} h)_{m}$ and $P_{m}$ as calculated from eqns (8) and (9). Fig 4 (right) shows the relation between $(\bar{D} h)_{m}$ and $P_{m}$, the lines are joining the average value of $(D h)_{m}$ for each value of $P_{m}$. Fig 4 suggests the possibility that within the normal physiological range the active force can be expressed in the linear form $(D h)_{m} \approx \mathrm{a} P_{m}+\mathrm{b}$. 
Table 1: $\quad$ Calculation of $(D h-P)_{m}$ and $(D h)_{m}$ by using eqn (7).

\begin{tabular}{|lccccccc|}
\hline $\mathrm{r} \mathrm{cm}$ & 1.44 & 1.34 & 1.236 & 1.14 & 1.036 & 0.94 & 0.84 \\
$50 \mathrm{mmHg}$ PP & & & & & & \\
$P_{m}=59.17 \mathrm{mmHg}$ & & & & & & \\
$\sigma_{\text {tot }}$ & -26.7 & -43.0 & -47.7 & -56.9 & -60.0 & -75.4 & -84.4 \\
$(D h-P)_{m}$ & 37.53 & 56.23 & 56.82 & 60.98 & 55.94 & 60.20 & 55.57 \\
$(D h)_{m}$ & 96.7 & 115.4 & 115.9 & 120.15 & 115.1 & 119.4 & 114.7 \\
\hline $75 \mathrm{mmHg}$ PP & & & & & & \\
$P_{m}=77 \mathrm{mmHg}$ & & & & & & \\
$\sigma_{t o t}$ & -33.3 & -57.8 & -64.6 & -77.8 & -80.7 & -108.1 & -107.4 \\
$(D h-P)_{m}$ & 46.80 & 75.58 & 76.95 & 83.38 & 75.24 & 86.3 & 70.71 \\
$(D h)_{m}$ & 123.8 & 152.6 & 153.9 & 160.4 & 152.2 & 163.3 & 147.7 \\
\hline $100 \mathrm{mmHg} \mathrm{PP}$ & & & & & & \\
$P_{m}=100 \mathrm{mmHg}$ & & & & & & \\
$\sigma_{t o t}$ & -38.5 & -68.1 & -73.1 & -96.9 & -104.6 & -128.9 & -134.8 \\
$(D h-P)_{m}$ & 54.11 & 89.05 & 87.07 & 103.85 & 97.52 & 102.91 & 88.75 \\
$(D h)_{m}$ & 154.1 & 189.1 & 187.1 & 203.9 & 197.5 & 202.9 & 188.7 \\
\hline
\end{tabular}

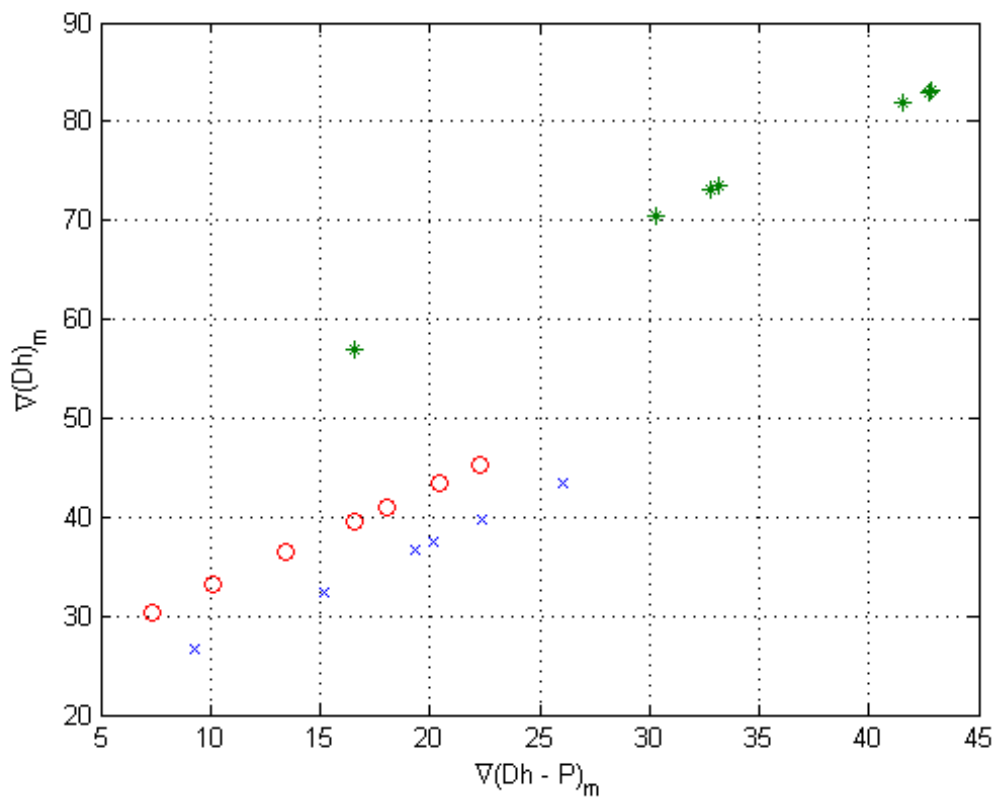

Figure 3: Relation between the variations in the active force $(D h)_{m}$ generated on the endocardium and the force $(D h-P)_{m}$ acting on the same surface of the myocardium as calculated from eqn (7) for three perfusion pressures as reported in Table 1. 

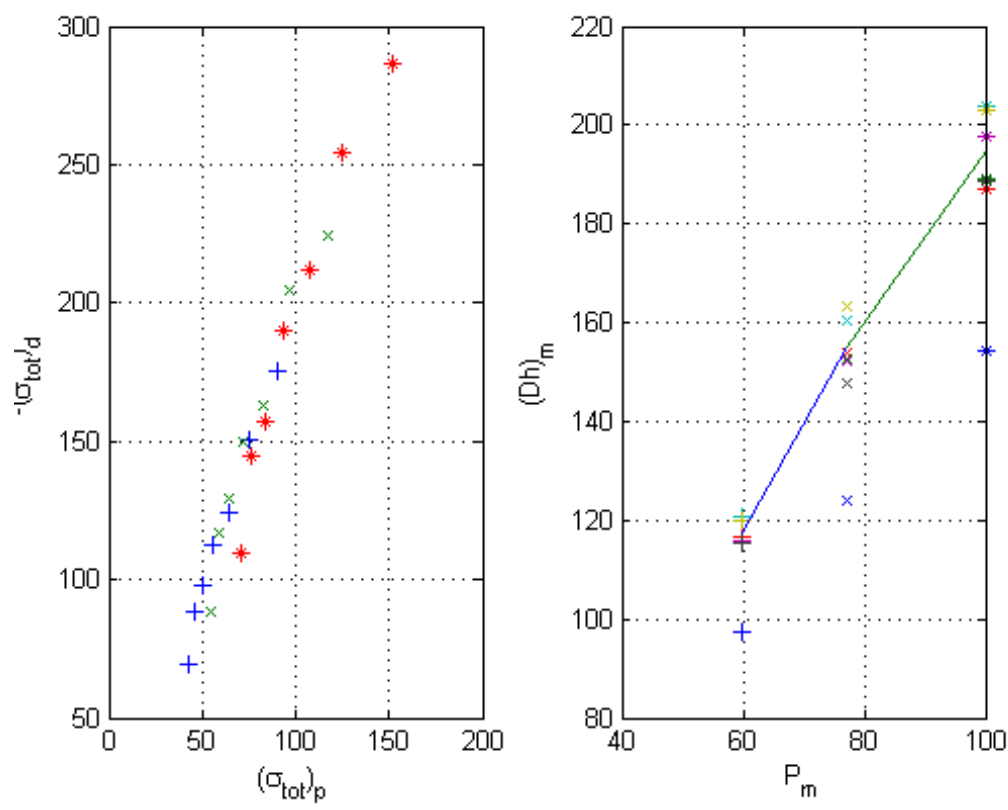

Figure 4: Left: Graphical relations between the components of total stress induced in the passive medium of the myocardium by $P_{m}$ and $(D h)_{m}$ for three perfusion pressures (see eqns (7-9)). Right: Relation between the active force $(D h)_{m}$ generated on the endocardium and the left ventricular pressure $P_{m} \approx P_{\max } / 1.2$.

\section{Conclusion}

The application of the linear theory of elasticity in the past has resulted in conflicting results depending of the approximations used. The present method in which the active force generated by the fibers in the myocardium is modelled as a force $D$ generated by unit volume of the myocardium has shown that the linear theory of elasticity can give consistent results. An interesting possibility that needs further study is that the active force/unit area $(D h)_{m}$ generated on the endocardium can be expressed within the physiological range as a linear relation to the left ventricular pressure $P_{m}$.

\section{References}

[1] Porter, W.T., The influence of heart beat on the flow of blood through the wall of the heart, American Journal of Physiology 1, pp. 145-163, 1898.

[2] Johnson, D.R. \& DiPalma, J.R., Intramyocardial pressure and its relation to aortic blood pressure, American Journal of Physiology 125, pp. 234-243, 1939. 
[3] Nematzadeh, D., Rose, J.C., Schryver, T., Huang, H.K. \& Kot, P.A., Analysis of the methodology foe measurement of intramyocardial pressure, Basic Research in Cardiology 79: 86-97, 1984.

[4] Mihailescu, L.S. \& Abel, F.L., Intramyocardial pressure gradients in working and nonworking isolated cat hearts, American Journal of Physiology 266, pp. H1233-H1241, 1994.

[5] Shoucri, R.M., Theoretical study of pressure-olume relation in left ventricle, American Journal of Physiology 260, pp. H282-H291, 1991.

[6] Shoucri, R.M., Possible clinical applications of the external work reserve of the myocardium, Japanese Heart Journal 35, pp. 771-787, 1994.

[7] Shoucri, R.M., Studying the mechanics of left ventricular contraction, IEEE Engineering in Medicine and Biology Magazine 17(3), pp. 95-101, May/June 1998.

[8] Shoucri, R.M., Active and passive stresses in the myocardium, American Journal of Physiology 279, pp. H2519-H2528, 2000.

[9] Shoucri, R.M., The calculation of the intramyocardial stress, Technology \& Health Care 10, pp. 11-22, 2002.

[10] Shoucri, R.M., The intramyocardial stress and the mechanics of the left ventricular contraction, Simulations in Biomedicine V, eds. Z.M. Arnez, C.A. Brebbia, F. Solina \& V. Stankovski, WIT Press: Southampton and Boston, pp. 177-187, 2003.

[11] Southwell, R.V., An Introduction to the Theory of Elasticity, Dover: New York, 1969.

[12] McHale, P.A. \& Greenfeld, J.C., Evaluation of several geometric models for estimation of left ventricular circumferential wall stress, Circulation Research 33, pp. 303-312, 1973. 